\title{
HARDY MARTINGALES AND JENSEN'S INEQUALITY
}

\author{
Nakhlé H. Asmar and Stephen J. Montgomery-Smith
}

\begin{abstract}
Hardy martingales were introduced by Garling and used to study analytic functions on the $N$-dimensional torus $\mathbb{T}^{N}$, where analyticity is defined using a lexicographic order on the dual group $\mathbb{Z}^{N}$. We show how, by using basic properties of orders on $\mathbb{Z}^{N}$, we can apply Garling's method in the study of analytic functions on an arbitrary compact Abelian group with an arbitrary order on its dual group. We illustrate our approach by giving a new and simple proof of a famous generalised Jensen's Inequality due to Helson and Lowdenslager [5].
\end{abstract}

\section{INTRODUCTION}

Suppose that $G$ is a nonzero compact connected Abelian group with an infinite (torsion-free) dual group $\Gamma$, and normalised Haar measure $\lambda$. For $1 \leqslant p<\infty$, the Banach space of measurable functions $f$ such that $|f|^{p}$ is integrable will be denoted by $L^{p}(G)$, and the Banach space of essentially bounded measurable functions on $G$ will be denoted by $L^{\infty}(G)$. We use the symbols $\mathbb{N}, \mathbb{Z}, \mathbb{R}$, and $\mathbb{C}$ to denote the natural numbers, the integers, the real numbers, and the complex numbers respectively. The circle group will be denoted by $\mathbb{T}$ and will be parametrised as $\left\{e^{i t}: 0 \leqslant t<2 \pi\right\}$.

A subset $P$ of $\Gamma$ is called an order if it satisfies the following three axioms:

$$
P \cap(-P)=\{0\}, P \cup(-P)=\Gamma, \text { and } P+P=P .
$$

Given an order $P \subset \Gamma$, we define a signum function with respect to $P$, $\operatorname{sgn}_{P}$, by: $\operatorname{sgn}_{P}(\chi)=-1,0$, or 1 , according as $\chi \in(-P) \backslash\{0\}, \chi=0$, or $\chi \in P \backslash\{0\}$. A function $f \in L^{1}(G)$ is called analytic if its Fourier transform $\hat{f}$ vanishes off $P$. This notion of analyticity was introduced by Helson and Lowdenslager [5] and [6] in connection with prediction theory, and since then it has been extensively studied because of its independent interest. For $1 \leqslant p \leqslant \infty$, following [6], we let $H^{p}(G)$ denote the space of analytic functions in $L^{p}(G)$. To be specific about the order, we shall also use the notation $H_{P}^{p}(G)$.

Received 20th March, 1996.

The work of the authors was supported by separate grants from the National Science Foundation (U.S.A.).

Copyright Clearance Centre, Inc. Serial-fee code: 0004-9729/97 \$A2.00+0.00. 
Recently, Garling [3] introduced Hardy martingales and used them in [4] to prove various properties of analytic functions on $\mathbb{T}^{N}$, the $N$-dimensional torus. (See Section 3 below for a review of these notions.) Analyticity in [4] was defined with respect to the following lexicographic order on the dual group $\mathbb{Z}^{N}$ :

$$
\begin{aligned}
P^{*}= & \{0\} \cup\left\{\left(m_{1}, m_{2}, \ldots, m_{N}\right) \in \mathbb{Z}^{N}: m_{1}>0, m_{2}=\ldots=m_{N}=0\right\} \\
& \cup\left\{\left(m_{1}, m_{2}, \ldots, m_{N}\right) \in \mathbb{Z}^{N}: m_{2}>0, m_{3}=\ldots=m_{N}=0\right\} \\
& \cup \ldots \cup\left\{\left(m_{1}, m_{2}, \ldots, m_{N}\right) \in \mathbb{Z}^{N}: m_{N}>0\right\} .
\end{aligned}
$$

Our goal in this paper is to show how Garling's approach in [4] can be applied in the setting of an arbitrary order on the dual group, by using basic properties of orders on $\mathbb{Z}^{N}$. We shall derive these properties in Section 2. In Section 3, we illustrate our approach by giving a simple proof of a generalised Jensen's Inequality for functions in $H^{1}(G)$, due to Helson and Lowdenslager [5]. The inequality states that for any $f \in H_{P}^{1}(G)$ we have

$$
\left|\int_{G} f(x) d \lambda(x)\right| \leqslant \exp \left(\int_{G} \log |f(x)| d \lambda(x)\right)
$$

This inequality plays an important role in the study of analytic measures on groups, factorisation of $H^{1}(G)$ functions, and the invariant subspace theory in $H^{2}(G)$ (see [5] and [6]). The only available proof of this inequality is the original one which uses the full strength of the methods in [5]. The proof that we offer is straightforward and follows directly from the corresponding inequality for functions in $H^{1}(\mathbb{T})$.

\section{ORDERS ON $\mathbb{Z}^{N}$}

Our goal in this section is to prove a useful property of orders which states that, given an arbitrary order $P$ on $\mathbb{Z}^{N}$ and a finite subset $E$ of $P$, there is an isomorphism of $\mathbb{Z}^{N}$ onto itself mapping $E$ into the lexicographic order $P^{*}$. We start by recalling from [7, Appendix A] definitions and properties of some special subsets of discrete groups. Throughout this section, $\Gamma$ denotes an infinite torsion-free Abelian group.

Definition 2.1: A finite subset $S=\left\{b_{1}, b_{2}, \ldots, b_{k}\right\} \subset \Gamma$ is independent if $0 \notin S$ and if $n_{1} b_{1}+n_{2} b_{2}+\ldots+n_{k} b_{k}=0$ implies that $n_{1}=n_{2}=\ldots=n_{k}=0$ (each $n_{j}$ is an integer). An infinite subset is called independent if every one of its finite subsets is independent. If an independent set $S$ generates $\Gamma$ then $S$ is called a basis. As a convention, if $B=\left\{b_{1}, b_{2}, \ldots\right\}$ is a basis in $\Gamma$ and $x \in \Gamma$, we shall write $b_{j}(x)$ for the $j$ th coordinate of $x$ in that basis. Hence $x=\sum_{j=1}^{k} b_{j}(x) b_{j}$, for some positive integer $k$. 
Definition 2.2: A subgroup $H$ of a torsion-free group $\Gamma$ is called pure in $\Gamma$ if whenever $x \in \Gamma, n \neq 0, n x \in H$, then $x \in H$.

Pure subgroups will arise in our proofs as kernels of homomorphisms. The concept of pure subgroups is very important in the study of the structure of Abelian groups. (See [7, Appendix A].) In what follows, we list some basic properties related to this concept, which will be needed in the sequel.

REMARK 2.3 .

(a) Suppose that $H$ and $K$ are subgroups of $\Gamma$, and that $H \subset K \subset \Gamma$. It is easy to see that if $K$ is pure in $\Gamma$ and $H$ is pure in $K$, then $H$ is pure in $\Gamma$.

The following is a very important property of pure subgroups of $\mathbb{Z}^{N}$.

(b) If $H$ is a proper pure subgroup of $\mathbb{Z}^{N}$, then $H$ is isomorphic to $\mathbb{Z}^{\nu}$ for some $\nu<N$. In this case, there is a basis of $\mathbb{Z}^{N}$ of the form $\left\{b_{1}, b_{2}, \ldots, b_{\nu}, \ldots, b_{N}\right\}$ such that $\left\{b_{1}, b_{2}, \ldots, b_{\nu}\right\}$ is a basis of $H$ (see [7, Theorem A. 26]). The number $\nu$ is called the rank, or the dimension, of $H$, and will be denoted $r(H)$.

Using (a), we can generalise (b) as follows.

(c) If $\{0\} \subset H_{1} \subset H_{2} \subset \ldots \subset H_{k}=\mathbb{Z}^{N}$ is a sequence of subgroups of $\mathbb{Z}^{N}$ such that $B_{j}$ is a proper pure subgroup of $H_{j+1}$, then there is a basis of $\mathbb{Z}^{N}, B=\left\{b_{1}, \ldots, b_{r\left(H_{1}\right)}, \ldots, b_{r\left(H_{j}\right)}, \ldots, b_{r\left(H_{k}\right)}\right\}$, such that, for $j=1, \ldots, k,\left\{b_{1}, \ldots, b_{r\left(H_{j}\right)}\right\}$ is a basis of $H_{j}$.

In addition to these notions, we need the following result, [1, Theorem (2.5)], that describes orders in $\mathbb{Z}^{N}$ in terms of a decreasing sequence of subgroups and corresponding separating real-valued homomorphisms.

Theorem 2.4. Let $P$ be an arbitrary order on $\mathbb{Z}^{N}$. There are a strictly increasing sequence of subgroups

$$
\{0\}=C_{0} \subset C_{1} \subset \ldots \subset C_{k-1} \subset C_{k}=\mathbb{Z}^{N},
$$

and a sequence $\left\{L_{j}\right\}_{j=1}^{k}$ of real-valued homomorphisms of $\mathbb{Z}^{N}$ such that, for $j=$ $1,2, \ldots, k$, we have

(i) $L_{j}\left(C_{j-1}\right)=\{0\}$;

(ii) $\operatorname{sgn}_{P}(\chi)=\operatorname{sgn}\left(L_{j}(\chi)\right)$, for all $\chi \in C_{j} \backslash C_{j-1}$.

For the lexicographic order $P^{*}$ (see Section 1), Theorem 2.4 is obvious. In this case, we have $k=N ; C_{0}=\{0\}, C_{1}=\left\{x \in \mathbb{Z}^{N}: x_{2}=\ldots=x_{N}=0\right\}, \ldots, C_{N-1}=$ $\left\{x \in \mathbb{Z}^{N}: x_{N}=0\right\}, C_{N}=\mathbb{Z}^{N} ;$ and $L_{j}\left(x_{1}, x_{2}, \ldots, x_{N}\right)=x_{j}$ for $j=1, \ldots, N$. 
Since $C_{k-1}$ is the kernel of a homomorphism of $\mathbb{Z}^{N}$, it follows immediately that $C_{k-1}$ is a pure subgroup of $\mathbb{Z}^{N}$. Similarly, $C_{k-2}$ is a pure subgroup of $C_{k-1}$. Now by Remark 2.3 (a), it follows that $C_{k-2}$ is a pure subgroup of $\mathbb{Z}^{N}$. Continuing in this fashion, we obtain the following simple proposition.

Proposition 2.5. In the notation of Theorem 2.4, we have that every $C_{j}$, $j=1, \ldots, k$, is a pure subgroup of $\mathbb{Z}^{N}$.

Let $H$ denote a nonzero pure subgroup of $\mathbb{Z}^{N}$. Write an element $x \in H$ as $x=\sum_{l=1}^{r(H)} b_{l}(x) b_{l}$ where $\left\{b_{l}\right\}_{l=1}^{r(H)}$ is a basis of $H$. A nonzero homomorphism $L$ of $H$ into $\mathbb{R}$ is said to have integer coefficients if

$$
L(x)=\sum_{l=1}^{r(H)} \alpha_{l} b_{l}(x)
$$

where $\left\{\alpha_{l}\right\}_{l=1}^{r(H)} \subset \mathbb{Z}$. Note that if $L$ has integer coefficients with respect to one basis, then it has integer coefficients with respect to all bases.

LEMMA 2.6. Suppose that $H \neq\{0\}$ is a pure subgroup of $\mathbb{Z}^{N}$, and $L$ is a nonzero homomorphism of $H$ with integer coefficients. Let $K=\operatorname{ker} L=\{x \in H$ : $L(x)=0\}$. Then there is an element $h \neq 0 \in B$ such that

$$
\boldsymbol{H}=K \oplus\langle h\rangle,
$$

where $\langle h\rangle$ denotes the subgroup of $\mathbb{Z}^{N}$ generated by $h$. In other words, $\operatorname{ker} L$ has codimension 1 in $B$.

Proof: Since $H$ is a pure subgroup, we may without loss of generality assume that $H \equiv \mathbb{Z}^{\nu}$. The homomorphism $L$ may be considered as a homomorphism from the linear vector space $\mathbb{Q}^{\nu}$ (over the field $\mathbb{Q}$ ) into $\mathbb{Q}$. Since the rank of this mapping is clearly 1 , its kernel has dimension $\nu-1$, and so contains $\nu-1$ independent vectors over $\mathbb{Q}$. By multiplying these vectors by a large enough integer, we get $\nu-1$ independent vectors of $\mathbb{Z}^{\nu}$ belonging to $\operatorname{ker} L$. These vectors necessarily form a basis for $K$. Now, since ker $L$ is a pure subgroup of $\mathbb{Z}^{\nu}$, the lemma follows from Remark 2.3(c).

Some more items of notation are needed before we state the main theorem of this section. To be specific about an order $P$ on a group $\Gamma$, we shall sometimes write $(\Gamma, P)$. Given a nonvoid finite subset $S$ of $\left(\mathbb{Z}^{N}, P\right)$, we shall write $S_{j}$ to denote the set

$$
S_{j}=S \cap\left(C_{j} \backslash C_{j-1}\right), \quad j=1,2, \ldots, k,
$$

where $C_{j}$ is the subgroup of $\mathbb{Z}^{N}$ given by Theorem 2.4. According to Theorem 2.4, there are at most $k \leqslant N$ such sets $S_{j}$, uniquely determined by $P$. The case of a 
lexicographic order is particularly interesting to us. In that case, we shall use the notation $S_{j}^{*}$ for the sets $S_{j}$. Hence, for $j=1, \ldots, N$, we have

$$
S_{j}^{*}=\left\{x \in S: x_{j} \neq 0, x_{j+1}=\ldots=x_{N}=0\right\}
$$

THEOREM 2.7. Let $S$ be a finite nonvoid subset of $\mathbb{Z}^{N}$, let $P$ be an arbitrary order on $\mathbb{Z}^{N}$, and let $\left\{C_{j}\right\}_{j=0}^{k}$ be as in Theorem 2.4. There is an isomorphism

$$
\psi:\left(\mathbb{Z}^{N}, P\right) \longrightarrow\left(\mathbb{Z}^{N}, P^{*}\right)
$$

such that $\psi(S \cap P) \subset P^{*}$, and $\psi(S \cap(-P)) \subset\left(-P^{*}\right)$. Moreover, for $j=1, \ldots, k$, we have

$$
\psi\left(S_{j}\right)=(\psi(S))_{r\left(C_{j}\right)}^{*}
$$

Proof: We apply Theorem 2.4 and use its notation. We shall construct a basis $B$ of $\mathbb{Z}^{N}$ of the form

$$
B=\left\{c_{1}, \ldots, c_{r\left(C_{1}\right)}, \ldots, c_{r\left(C_{j}\right)}, \ldots, c_{r\left(C_{k}\right)}\right\}
$$

so that, in that basis, every element of $S_{j}$ has a nonzero $r\left(C_{j}\right)$-th component, and $x \in S_{j} \cap P$ if and only if $x \in S_{j}$ and $c_{r\left(c_{j}\right)}(x)>0$. Then the theorem will follow by setting

$$
\psi\left(\sum_{l=1}^{N} c_{l}(x) c_{l}\right)=\sum_{l=1}^{N} c_{l}(x) e_{l}
$$

where $\left\{e_{1}, \ldots, e_{N}\right\}$ denotes the standard basis in $\mathbb{Z}^{N}$. We now proceed to show how to construct $B$. Without loss of generality, we may assume that $S_{j}$ is not empty for all $j=1, \ldots, k$. Let

$$
B_{1}=\left\{b_{1}, \ldots, b_{r\left(C_{1}\right)}, \ldots, b_{r\left(c_{j}\right)}, \ldots, b_{r\left(C_{k}\right)}\right\}
$$

denote a basis of $\mathbb{Z}^{N}$ with the property that $\left\{b_{1}, \ldots, b_{r\left(C_{1}\right)}, \ldots, b_{r\left(C_{j}\right)}\right\}$ is a basis for $C_{j}$ (Remark 2.3(c)). For $x \in \mathbb{Z}^{N}$, we write $x=\sum_{l=1}^{N} b_{l}(x) b_{l}$. Expressing the homomorphism $L_{j}$ in the basis $B_{1}$, we have for $x \in C_{j}$,

$$
L_{j}(x)=\sum_{l=r\left(C_{j-1}\right)+1}^{r\left(C_{j}\right)} \beta_{l} b_{l}(x)
$$


where $\beta_{l} \in \mathbb{R}$, because $L_{j}\left(C_{j-1}\right)=\{0\}$. Since, by Theorem 2.4(ii), $L_{j}(x)>0$ for all $x \in S_{j} \cap P$, and $L_{j}(x)<0$ for all $x \in S_{j} \cap(-P)$, and since $S$ is finite, we can replace the coefficients $\beta_{l}$ in (3) by integers $\alpha_{l}$ so that

$$
\sum_{l=r\left(C_{j-1}\right)+1}^{r\left(C_{j}\right)} \alpha_{l} b_{l}(x)>0
$$

if $x \in S_{j} \cap P$, and

$$
\sum_{l=r\left(C_{j-1}\right)+1}^{r\left(C_{j}\right)} \alpha_{l} b_{l}(x)<0
$$

if $x \in S_{j} \cap(-P)$. (First replace the real numbers $\alpha_{l}$ in (3) by rational numbers, then multiply by a sufficiently large positive integer.) Define a homomorphism $L_{j}^{*}$ for all $x \in \mathbb{Z}^{N}$ by

$$
L_{j}^{*}(x)=\sum_{l=r\left(C_{j-1}\right)+1}^{r\left(C_{j}\right)} \alpha_{l} b_{l}(x) .
$$

Plainly, $L_{j}^{*}(x) \neq 0$, for all $x \in S_{j}$. Let $D_{j}=\left\{x \in C_{j}: L_{j}^{*}(x)=0\right\}$. Then $D_{j} \supset C_{j-1}$, and, by Lemma 2.6, $D_{j}$ has codimension 1 in $C_{j}$. Let $h_{j} \in C_{j}$ be such that

$$
C_{j}=D_{j} \oplus\left\langle h_{j}\right\rangle .
$$

We can and do choose $h_{j}$ so that $L_{j}^{*}\left(h_{j}\right)>0$. Now consider the basis

$$
B=\left\{c_{1}, \ldots, c_{r\left(D_{1}\right)}, h_{1}=c_{r\left(C_{1}\right)}, \ldots, c_{r\left(D_{j}\right)}, h_{j}=c_{r\left(C_{j}\right)}, \ldots, c_{r\left(D_{k}\right)}, h_{k}=c_{r\left(C_{k}\right)}\right\}
$$

where $\left\{c_{1}, \ldots, c_{r\left(D_{1}\right)}, h_{1}, \ldots, c_{r\left(D_{j}\right)}\right\}$ is a basis for $D_{j}$, and hence

$$
\left\{c_{1}, \ldots, c_{r\left(D_{1}\right)}, h_{1}, \ldots, c_{r\left(D_{j}\right)}, h_{j}\right\}
$$

is a basis for $C_{j}$. For $x \in S_{j}$ we have $L_{j}^{*}(x) \neq 0$, and so $h_{j}(x) \neq 0$. Also, $x \in S_{j} \cap P$ if and only if $x \in S_{j}$ and $L_{j}^{*}(x)>0$, if and only if $x \in S_{j}$ and $h_{j}(x)>0$. This shows that $B$ has the desired properties and completes the proof of the theorem.

\section{HaRdy Martingales and JENSEN'S INEQuality}

We start this section by reviewing the concept of Hardy martingales from [4]. Let $N$ be a fixed positive integer, and let $e^{i \theta_{n}}$ denote the $n$-th coordinate evaluation function on $\mathbb{T}^{N}$. Let $\mathcal{F}_{n}=\sigma\left(e^{i \theta_{1}}, e^{i \theta_{2}}, \ldots, e^{i \theta_{n}}\right)$ denote the $\sigma$-algebra generated by 
the first $n$ coordinate functions. For $f \in L^{1}\left(\mathbb{T}^{N}\right)$, the conditional expectation of $f$ with respect to $\mathcal{F}_{n}$ will be denoted $\mathbb{E}\left(f \mid \mathcal{F}_{n}\right)$. For $n=0,1,2, \ldots, N$, the function $\mathbb{E}\left(f \mid \mathcal{F}_{n}\right)$ is constructed from $f$ by projecting the Fourier transform of $f$ on $\mathbb{Z}^{n}$, where here $\mathbb{Z}^{0}=\{0\}$, and

$$
\mathbb{Z}^{n}=\left\{\left(k_{1}, k_{2}, \ldots, k_{n}, 0, \ldots, 0\right): k_{j} \in \mathbb{Z}\right\}
$$

The finite sequence $\left(\mathbb{E}\left(f \mid \mathcal{F}_{n}\right)\right)_{n=1}^{N}$ forms a martingale relative to $\left(\mathcal{F}_{n}\right)_{n=1}^{N}$. (A detailed analysis of martingales on groups with a decreasing sequence of subgroups can be found in [2, Chapter 5].) A complex-valued martingale $\left(f_{n}\right)$ on $\mathbb{T}^{N}$ is called a Hardy martingale if $\mathbb{E}\left(f_{n+1} e^{i k \theta_{n+1}} \mid \mathcal{F}_{n}\right)=0$ for $k>0$ and all $n=0, \ldots, N-1$.

Hardy martingales arise naturally when studying analytic functions in $H^{1}\left(\mathbb{T}^{N}\right)$. Indeed, as observed in [4], for $1 \leqslant p \leqslant \infty$, we have

$$
H_{P^{*}}^{p}\left(\mathbb{T}^{N}\right)=\left\{f \in L^{p}\left(\mathbb{T}^{N}\right):\left(\mathbb{E}\left(f \mid \mathcal{F}_{n}\right)\right)_{n=1}^{N} \text { is a Hardy martingale }\right\}
$$

where here, as in Section 1, $P^{*}$ denotes the lexicographic order on $\mathbb{Z}^{N}$.

It is instructive to justify this fact, and show, in the process, how Hardy martingales are related to the usual Hardy spaces on the circle group. For this purpose, we recall the notion of martingale differences series. Let

$$
d_{0}(f)=\int_{\mathbb{T}^{N}} f d x
$$

and for $j=1, \ldots, N$, let

$$
d_{j}(f)=\mathbb{E}\left(f \mid \mathcal{F}_{j}\right)-\mathbb{E}\left(f \mid \mathcal{F}_{j-1}\right) .
$$

Thus $d_{j}(f)(j=1, \ldots, N)$ is constructed from $f$ by projecting the Fourier transform of $f$ on the set difference $\mathbb{Z}^{j} \backslash \mathbb{Z}^{j-1}$. So $d_{j}(f)$ may be formally represented as the Fourier series:

$$
d_{j}(f)=\sum_{k=-\infty, k \neq 0}^{\infty} f_{j, k}\left(\theta_{1}, \ldots, \theta_{j-1}\right) e^{i k \theta_{j}},
$$

where $f_{j, k}\left(\theta_{1}, \ldots, \theta_{j-1}\right)$ is a function of $\theta_{1}, \ldots, \theta_{j-1}$ only. We have the martingale difference series decomposition

$$
f=\sum_{j=0}^{N} d_{j}(f)
$$


As observed in [4], and is easy to check, $f$ is in $H_{P^{*}}^{1}\left(\mathbb{T}^{N}\right)$ if and only if, for $j=$ $1, \ldots, N$,

$$
d_{j}(f)=\sum_{k=1}^{\infty} f_{j, k}\left(\theta_{1}, \ldots, \theta_{j-1}\right) e^{i k \theta_{j}} .
$$

Hence, as a function of $\theta_{j}$, the function $d_{j}(f)$ belongs to $H^{1}(\mathbb{T})$ for $j=1,2, \ldots, N$. From these observations, (4) follows easily.

We now return to inequality (1), and prove a special case of it.

Proposition 3.1. Suppose that $f \in H_{P^{*}}^{1}\left(\mathbb{T}^{N}\right)$. Then,

$$
\exp \left(\int_{\mathbb{T}^{N}} \log \left|\sum_{j=0}^{n} d_{j}(f)\right| d x\right) \leqslant \exp \left(\int_{\mathbb{T}^{N}} \log \left|\sum_{j=0}^{n+1} d_{j}(f)\right| d x\right)
$$

and

$$
\left|\int_{\mathbb{T}^{N}} f d x\right| \leqslant \exp \left(\int_{\mathbb{T}^{N}} \log |f| d x\right) .
$$

Proof: The second inequality follows by applying the first inequality repeatedly (for $n=0,1, \ldots, N-1$ ) and using (5) and (7). Let us prove the first inequality. As we observed above, the function $\theta_{n+1} \mapsto d_{n+1}(f)$ is in $H^{1}(\mathbb{T})$. Using the one-dimensional version of Jensen's Inequality (1) for functions in $H^{1}(\mathbb{T})$ (see [10, Inequality (3.2), and Theorem 3.11]), we obtain

$$
\log \left|\frac{1}{2 \pi} \int_{\theta_{n+1}}\left(\sum_{j=0}^{n+1} d_{j}(f)\right) d \theta_{n+1}\right| \leqslant \frac{1}{2 \pi} \int_{\theta_{n+1}} \log \left|\sum_{j=0}^{n+1} d_{j}(f)\right| d \theta_{n+1} .
$$

But

$$
\int_{\theta_{n+1}} d_{n+1}(f) d \theta_{n+1}=0
$$

and $d_{j}(f)$ is constant in $\theta_{n+1}$ for $j=0,1, \ldots, n$. Thus,

$$
\log \left|\sum_{j=0}^{n} d_{j}(f)\right| \leqslant \frac{1}{2 \pi} \int_{\theta_{n+1}} \log \left|\sum_{j=0}^{n+1} d_{j}(f)\right| d \theta_{n+1} .
$$

Integrating with respect to the remaining variables, we get

$$
\int_{\mathbb{T}^{N}} \log \left|\sum_{j=0}^{n} d_{j}(f)\right| d x \leqslant \int_{\mathbb{T}^{N}} \log \left|\sum_{j=0}^{n+1} d_{j}(f)\right| d x,
$$

which completes the proof of the proposition.

For the remainder of the proof, we need the following density result. 
Lemma 3.2. Let $G$ be a compact Abelian group with dual ordered by $P$, and let $Y$ be a dense subspace of $H_{P}^{1}(G)$. If (1) is true for all $f$ in $Y$, then (1) is true for all $f$ in $H_{P}^{1}(G)$.

Proof: We first note, for a given function $f \in L^{1}(G)$, that (1) holds if and only if for all $0<p<1$ we have

$$
\left|\int_{G} f d \lambda\right|^{p} \leqslant \int_{G}|f|^{p} d \lambda
$$

Indeed (1) follows from (9) by letting $p$ tend to zero (see $[\mathbf{9 , ( 1 3 . 3 2 ) ( i i ) ] ) . ~ N o w ~ s u p p o s e ~}$ that (1) holds. Then, for any $0<p<1$, we have

$$
\left|\int_{G} f d \lambda\right|^{p} \leqslant \exp \left(\int_{G} \log \left(|f|^{p}\right) d \lambda\right) \leqslant \int_{G}|f|^{p} d \lambda,
$$

where the last inequality follows from $[9,(13.32)(i)]$.

Now fix $0<p<1$ and $f \in H_{P}^{1}(G)$. Let $\left(f_{n}\right)$ be a sequence in $Y$ such that $f_{n} \rightarrow f$ in $L^{1}(G)$. We have that $f_{n} \rightarrow f$ in $L^{p}$, and from the inequality $\left.\int_{G}|| f_{n}\right|^{p}-|f|^{p} \mid d \lambda \leqslant$ $\int_{G}\left|f_{n}-f\right|^{p} d \lambda$, it follows that $\int_{G}\left|f_{n}\right|^{p} d \lambda \rightarrow \int_{G}|f|^{p} d \lambda$ (see [9, Theorem (13.17) and (13.25) (a)]). Since (9) holds for every $f_{n}$, it follows immediately that it also holds for $f$.

The next step is to establish (1) for arbitrary orders on $\mathbb{Z}^{N}$. At this point we shall appeal to Theorem 2.7 .

Proposition 3.3. Suppose that $f \in H_{P}^{1}\left(\mathbb{T}^{N}\right)$. Then,

$$
\left|\int_{\mathbb{T}^{N}} f d x\right| \leqslant \exp \left(\int_{\mathbb{T}^{N}} \log |f| d x\right) .
$$

Proof: Lemma 3.2 shows that it is enough to consider the case of a trigonometric polynomial $f \in B_{P}^{1}\left(\mathbb{T}^{N}\right)$. Write

$$
f=\sum_{\chi \in S} a_{\chi} \chi
$$

where $a_{\chi} \in \mathbb{C}$, and $S$ is a finite subset of $P$. Apply Theorem 2.7 and obtain an isomorphism $\psi$ of $\mathbb{Z}^{N}$ such that $\psi(S) \subset P^{*}$. Let $\phi$ be the adjoint homomorphism of $\psi$. Thus $\phi$ is an automorphism of $\mathbb{T}^{N}$ onto itself such that

$$
\psi(\chi)(x)=\chi \circ \phi(x)
$$

for all $\chi \in \mathbb{Z}^{N}$ and all $x \in \mathbb{T}^{N}$. Moreover, for all $\chi \in S$, the character $\chi \circ \phi=\psi(\chi)$ is in $P^{*}$. Since $\phi$ is an automorphism of $\mathbb{T}^{N}$, it maps the normalised Haar measure to 
itself. Using this last observation and Proposition 3.1, we obtain

$$
\begin{aligned}
\log \left|\int_{\mathbb{T}^{N}} f d x\right| & =\log \left|\int_{\mathbb{T}^{N}} f \circ \phi d x\right|=\log \left|\int_{\mathbb{T}^{N}} \sum_{\chi \in S} a_{\chi} \psi(\chi) d x\right| \\
& \leqslant \int_{\mathbb{T}^{N}} \log \left|\sum_{\chi \in S} a_{\chi} \psi(\chi)\right| d x=\int_{\mathbb{T}^{N}} \log |f| d x,
\end{aligned}
$$

which completes the proof of the proposition.

We are only a step away from completing the proof of (1). The standard reduction that remains to be done is based on the Weil formula. We present the details for the sake of completeness.

Proof of (1): Throughout this proof, $P$ will denote an arbitrary order on $\Gamma$. By Lemma 3.2, it is enough to consider trigonometric polynomials in $H_{P}^{1}(G)$. Let

$$
f=\sum_{\chi \in S} a_{\chi} \chi
$$

where $S$ is a nonvoid finite subset of $P$, and $a_{x} \in \mathbb{C}$. Let $\langle S\rangle$ denote the subgroup of $\Gamma$ generated by $S$. Since $\Gamma$ is torsion-free, $\langle S\rangle$ is isomorphic to $\mathbb{Z}^{N}$ for some positive integer $N$. Let $G_{0}$ denote the annihilator in $G$ of $\mathbb{Z}^{N}$. Then $G / G_{0}$ is topologically isomorphic to $\mathbb{T}^{N}$ and its dual group is $\langle S\rangle \equiv \mathbb{Z}^{N}$. We order $\mathbb{Z}^{N}$ by intersecting it with $P$. Let $I$ denote the natural homomorphism of $G$ onto $G / G_{0}$. Since $f$ is constant on cosets of $G_{0}$, there is a trigonometric polynomial on $\mathbb{T}^{N}, f^{\dagger}$, such that

$$
f=f^{\dagger} \text { ○ा }
$$

Clearly $f^{\dagger} \in H_{\mathbf{Z}^{N} \cap P}^{1}\left(\mathbb{T}^{N}\right)$. Now, using the Weil formula [8, Theorem (28.54) (iii)] and Proposition 3.3, we find that

$$
\begin{aligned}
\log \left|\int_{G} f d \lambda\right| & =\log \left|\int_{\mathbb{T}^{N}} f^{\dagger} d x\right| \\
& \leqslant \int_{\mathbb{T}^{N}} \log \left|f^{\dagger}\right| d x=\int_{G} \log |f| d \lambda
\end{aligned}
$$

which yields the desired inequality and completes the proof of (1).

\section{REFERENCES}

[1] N. Asmar, 'The conjugate function on the finite dimensional torus', Canad. Math. Bull. 32 (1989), 140-148. 
[2] R.E. Edwards and G.I. Gaudry, Littlewood-Paley and multiplier theory, Ergebnisse der Mathematik und ihrer Grenzgebiete 90 (Springer-Verlag, Berlin, Heidelberg, New York, 1977).

[3] D.J.H. Garling, 'On martingales with values in a complex Banach space', Math. Proc. Cambridge Philos. Soc. 104 (1988), 399-406.

[4] D.J.H. Garling, 'Hardy martingales and the unconditional convergence of martingales', Bull. London Math. Soc. 23 (1991), 190-192.

[5] H. Helson and D. Lowdenslager, 'Prediction theory and Fourier series in several variables', Acta Math. 99 (1958), 165-202.

[6] H. Helson and D. Lowdenslager, 'Prediction theory and Fourier series in several variables II', Acta Math. 106 (1961), 175-212.

[7] E. Hewitt and K.A. Ross, Abstract harmonic analysis $I$ ( $2^{\text {nd }}$ edition), Grundlehren der Math. Wissenschaften 115 (Springer-Verlag, Berlin, Heidelberg, New York, 1979).

[8] E. Hewitt and K.A. Ross, Abstract harmonic analysis II, Grundlehren der Math. Wissenschaften in Einzeldarstellungen 152 (Springer-Verlag, Berlin, Heidelberg, New York, 1970).

[8] E. Hewitt and K. Stromberg, Real and abstract analysis, Graduate Texts in Mathematics 25, (2nd printing) (Springer-Verlag, Berlin, Heidelberg, New York, 1969).

[10] Y. Katznelson, An introduction to harmonic analysis (John Wiley, New York, 1968).

\author{
Department of Mathematics \\ University of Missouri-Columbia \\ Columbia, MI 65211 \\ United States of America
}

